\title{
Physiotherapy interventions for increasing muscle strength in people with spinal cord injuries: a systematic review
}

\author{
Nisha Aravind ${ }^{1} \cdot$ Lisa A. Harvey (1) ${ }^{1} \cdot$ Joanne V. Glinsky ${ }^{1}$
}

Received: 28 June 2018 / Revised: 20 December 2018 / Accepted: 24 December 2018 / Published online: 5 February 2019

(c) International Spinal Cord Society 2019

\begin{abstract}
Study design A systematic review.

Objective The aim of this review was to determine the effectiveness of physiotherapy (PT) interventions for increasing voluntary muscle strength in people with spinal cord injuries (SCI).

Methods We included randomised controlled trials of PT interventions for people with SCI. We were interested in two comparisons: PT interventions compared with sham or no intervention, and PT interventions compared to each other. The outcome of interest was voluntary strength of muscles directly affected by SCI. All included studies were rated according to the Cochrane Risk of Bias Tool and results of similar trials were pooled using meta-analyses where possible.

Results Twenty-six trials met the inclusion criteria and provided useable data. A statistically significant between-group difference was found in four comparisons, namely, resistance training versus no intervention (standardised mean difference $(\mathrm{SMD})=0.64 ; 95 \% \mathrm{CI}, 0.22-1.07 ; p=0.003)$; resistance training combined with electrical stimulation versus no intervention (mean difference $(\mathrm{MD})=14 \mathrm{Nm}$; 95\% CI, 1-27; $p=0.03$ ); a package of PT interventions versus no intervention $(\mathrm{MD}=4.8 / 50$ points on the Lower Extremity Motor Score (LEMS); 95\% CI 1.9-7.7; $p=0.01$ ); and robotic gait training versus overground gait training (MD $=3.1 / 50$ points on the LEMS; 95\% CI, 1.3-5.0; $p=0.0008$ ).

Conclusion There is evidence that a small number of PT interventions increase voluntary strength in muscles directly affected by SCI.
\end{abstract}

\section{Introduction}

Physiotherapy (PT) interventions are widely used in an effort to increase the voluntary strength of muscles directly affected by spinal cord injury (SCI), that is, neurologicallyweak muscles. Some of the PT interventions commonly used for this purpose include resistance training [1], electrical stimulation [2, 3], hand therapy [4] and various types of gait training interventions [5-7]. It is important to better understand the effects of these interventions on strength because strength optimises function after SCI. In addition,

Supplementary information The online version of this article (https:// doi.org/10.1038/s41393-019-0242-z) contains supplementary material, which is available to authorised users.

Lisa A. Harvey

1.harvey@usyd.edu.au

1 John Walsh Centre for Rehabilitation Research, Sydney Medical School, University of Sydney, Sydney, Australia researchers investigating drug or cellular therapies for neurological recovery often want to know how routinely administered PT affects the strength of neurologically-weak muscles, particularly the key muscles that are part of the International Standards for the Neurological Classification of SCI [8-10].

Surprisingly little work has been directed at summarising what we know from clinical trials about the effectiveness of different PT interventions on voluntary strength of neurologically-weak muscles. Many systematic reviews (including some of our own [11-13]) have investigated the effectiveness of different interventions on mobility and function. Some of these have included strength as an outcome. However, no systematic review has solely focused on voluntary strength of neurologically-weak muscles and included all PT interventions. Therefore, the purpose of this systematic review was to provide an overview of the effectiveness of PT interventions for increasing voluntary strength of muscles directly affected by SCI.

The two primary objectives of this systematic review were to determine: 
1. The effectiveness of any PT intervention compared to sham or no intervention for increasing muscle strength in people with SCI. For example, the effectiveness of hand therapy versus no hand therapy on the grip strength of people with tetraplegia.

2. The relative effectiveness of any PT intervention compared to another PT intervention for increasing muscle strength in people with SCI. For example, the effectiveness of Lokomat gait training versus overground gait training on Lower Extremity Motor Scores (LEMS) of people with incomplete SCI.

\section{Methods}

We searched the following databases from inception to Jan 2018: Embase (via the Ovid search engine), Medline (via the Ovid search engine) and the Cochrane Central register of controlled trials. We also searched the Physiotherapy Evidence Database (PEDro). The Embase and Medline databases were searched using the Cochrane highly sensitive search strategy for identifying clinical trials [14] combined with the following $\mathrm{MeSH}$ terms: parapleg\$, quadripleg\$, tetrapleg\$, wheelchair\$ and spinal cord. We included a line in our search strategy to exclude animal studies. The searches were adjusted for each database. The Cochrane Central register of controlled trials was searched using the MeSH terms.

The searches were originally run in December 2007 [11], again in December 2015 [12], and then updated in Jan 2018. Two authors independently screened the titles and abstracts to generate a list of all randomised controlled trials (RCTs) investigating the effectiveness of any PT interventions involving people with SCI irrespective of outcomes, comparisons or purpose. As a second step, all three authors screened the list of RCTs to identify trials for this review. Full articles of any potentially eligible studies were retrieved and examined to check against the inclusion criteria. Any disagreements were resolved by further discussion.

\section{Inclusion criteria}

\section{Types of participants}

We included studies involving adults with a traumatic or non-traumatic SCI, and a complete or incomplete SCI regardless of time since injury. Trials involving participants with other conditions were only included if $80 \%$ or more of the participants had a SCI.

\section{Types of intervention}

We included studies which examined any type of PT intervention or combination of therapies provided the intervention required participants to voluntarily contract neurologically-weak muscles. This included any of the following administered with or without biofeedback, somatosensory stimulation or electrical stimulation:

1. Gait training such as robotic, overground and bodyweight supported treadmill training (BWSTT).

2. Hand therapy such as mass practice and practice provided using Wii-like devices.

3. Generic exercises and general fitness training programs such as arm cranking, hydrotherapy and home exercise programs.

4. Resistance training programs such as those typically involving free weights, theraband, strengthening equipment or any type of gym equipment.

We accepted studies with non-PT co-interventions including surgery, drug therapies or psychological interventions provided they were administered in exactly the same way to both groups. We reasoned that these cointerventions would have a similar effect across both groups thereby enabling us to isolate the effect of the PT treatment.

We excluded studies investigating the effectiveness of drug therapies, stem cell therapies, cranial stimulation, and studies that examined the effect of any intervention on the non-paralysed muscles of people with SCI (for example, studies that examined the effect of training programs on the upper limbs of people with paraplegia). Studies that included a combination of people with paraplegia and tetraplegia and involved training of the upper limbs, were excluded if $20 \%$ or more of participants had paraplegia. This was done in order to be confident that the effects of the training on the upper limbs predominantly reflected the effects on muscles directly affected by SCI. We also excluded studies that did not require participants to voluntarily contract neurologically-weak muscles as part of the intervention. Therefore, we excluded studies that examined interventions such as passive movements, passive cycling, vibration, transcutaneous electrical nerve stimulation (TENS), acupuncture or massage. The only exception to this last rule was any study which compared two or more interventions when at least one of the interventions required participants to voluntarily contract neurologically-weak muscles. For example, we excluded studies that compared stretching to no stretching but we included studies which compared stretching to robotic gait training. 


\section{Types of comparisons}

We were interested in two comparisons, namely:

(i) PT interventions compared to sham or no intervention.

(ii) PT interventions compared to each other.

If trials included more than two groups, we extracted data only from two groups. We prioritised the two groups which were most likely to demonstrate a between-group difference and were most commonly compared in other trials (namely, BWST versus overground gait training $[5,15]$, and conventional hand therapy plus somatosensory stimulation versus conventional hand therapy [16]).

\section{Types of outcomes}

Trials were only included if they assessed voluntary strength of muscles directly affected by SCI. That is, muscles that could be reasonably expected to have less than grade 5 strength (according to a manual muscle test). Strength could be measured in any way provided it reflected either force ( $\mathrm{kg}$ or Newtons), torque $(\mathrm{Nm})$ or results of a manual muscle test (points). We accepted composite measures of strength such as the LEMS (points). If studies included more than one measure to reflect voluntary strength, we selected the measure that was most often used by similar studies to enable pooling of data. If studies reported the strength of muscles on the left and right side of the body separately (e.g. right and left knee extensor strength), then we always extracted the measures from the right side of the body. If studies measured outcomes at more than one end point (e.g. measured at 6 weeks and 12 weeks from randomisation), then we used the outcomes reported as soon as possible after the last treatment. Two review authors independently identified the most relevant outcome and end-point from each trial according to our criteria. Any differences were resolved by the third author.

\section{Type of studies}

We only included RCTs and randomised cross-over trials that were published in English.

\section{Data extraction and synthesis}

Two authors independently extracted data from the included studies using a standardised Excel spreadsheet. One author was an investigator on some of the studies which were ultimately included in the review but the other author was not. The following descriptive data were extracted: characteristics of intervention and comparator, dosage, target muscle, study design, sample size, characteristics of participants, and details of strength measures used in the analysis.

Strength data were extracted from each study to determine mean between-group differences and $95 \%$ confidence intervals (95\% CI). Data were estimated from graphs if necessary. The following rule was used (from first to last preference) when deciding upon which data to extract:

- between-group difference in post-intervention scores, adjusted for baseline scores.

- mean and standard deviation (SD) of change scores (post-intervention scores and change scores were not pooled in meta-analyses in which results were expressed as standardised mean differences (SMD)).

- mean (SD) post-intervention scores.

If only medians and inter-quartile ranges (IQR) were provided, medians were extracted and used as means, and SDs were estimated by dividing the interquartile range by 1.35 [17]. Cross-over studies were analysed using combined data from all study periods, if available, or using first period data if combined data were not available. RevMan software [18] was used to convert 95\% CIs, standard errors, $p$ values and any other appropriate combination of data or statistical results into SDs when necessary. The direction of effect of each outcome was standardised.

Meta-analyses were conducted across studies that made similar comparisons if there were at least two studies without excessive clinical or statistical heterogeneity. Clinical heterogeneity was assessed by examining the type of participants, type and intensity of the intervention, and other issues related to the design and conduct of the studies. Statistical heterogeneity was quantified using the $I^{2}$ statistic where an $I^{2}>75 \%$ was considered to indicate excessive heterogeneity [17]. A fixed-effects model was used to pool data if the $I^{2}$ was less than $50 \%$, and a random-effects model was used if the $I^{2}$ was between 50 and $75 \%$. If studies in a meta-analysis used the same measure of strength and same units, effects were expressed as mean differences (MD) and 95\% CI. If different measures of strength or different units were used within a meta-analysis, effects were expressed as SMD and 95\% CI but post-interventions scores were not pooled with change scores. Data were analysed using RevMan v5.3 [18].

All studies were assessed for risk of bias using the following six items of the Cochrane Risk of Bias Tool: Random sequence generation (selection bias); Concealment of allocation (selection bias); Blinding of participants and personnel (performance bias); Blinding of assessors (detection bias); Incomplete outcome data (attrition bias); 


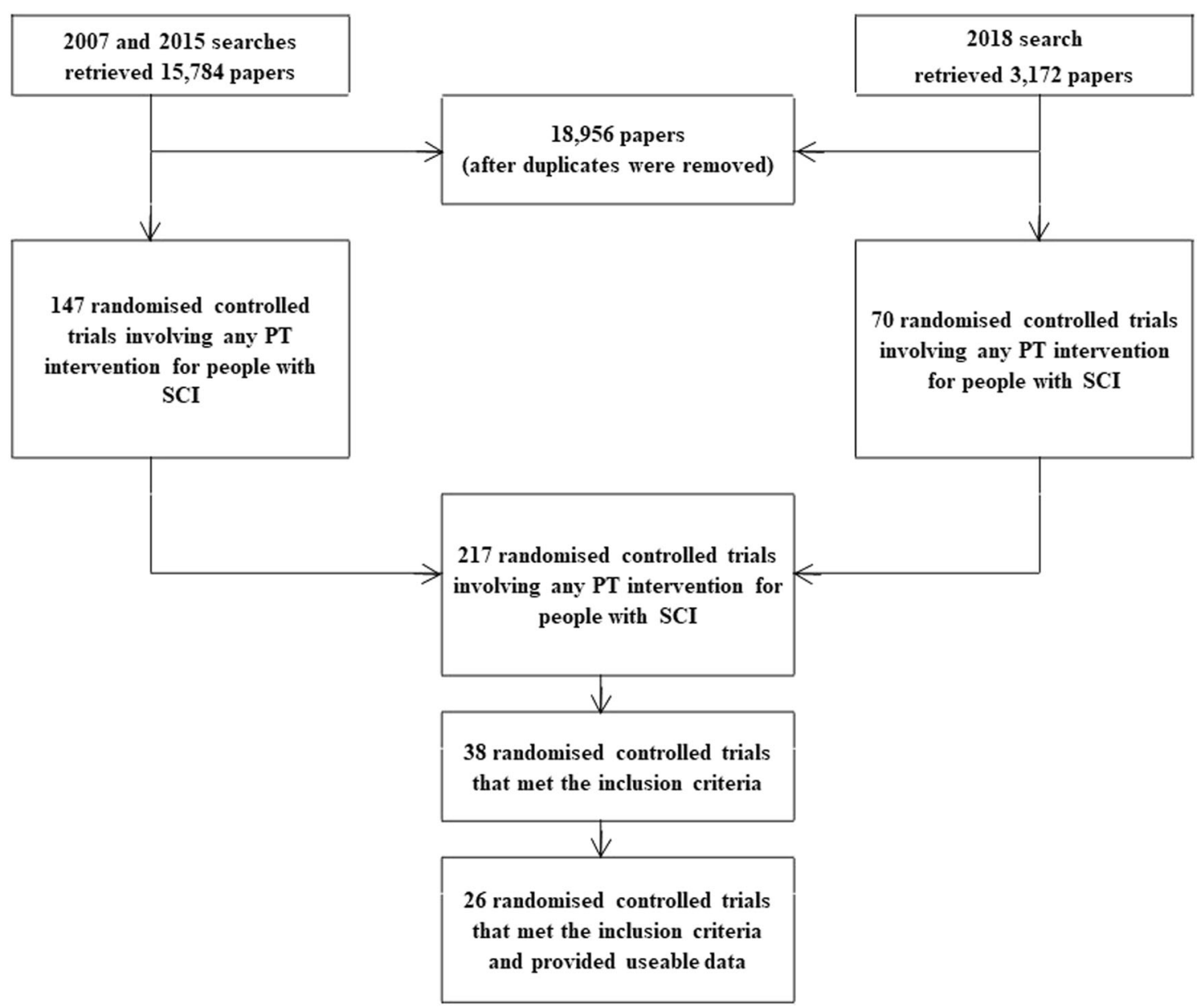

Fig. 1 Flow chart. PT Physiotherapy

and Selective reporting (reporting bias). Each item was rated as high, low or unclear risk of bias as per Cochrane guidelines [17].

\section{Results}

The searches from 2007, 2015 and Jan 2018 together retrieved 18,956 papers (see Fig. 1). From these, we identified 217 studies which were potentially RCTs in people with SCI involving a PT intervention. Nine trials which assessed the strength of the upper limbs were excluded because $20 \%$ or more of participants had paraplegia. Ultimately, after examining the full text and excluding duplicate publications, we identified 38 trials [1-7, 15, 16, 19-47] that met our inclusion criteria but only 26 provided useable data and hence were included [1, 2, 4-7, 15, 16, 19, 20, 22-27, 31-33, 35-37, 40, $43,44,46]$. The other 12 trials did not provide useable data: they stated that they measured strength but either did not report any strength data $[38,39,42,47]$ or did not provide sufficient data for inclusion in the forest plots [3, 21, 28-30, $34,41,45]$ (see the Supplementary File 1 for details).

The number of participants in each group in the 26 trials ranged from 5 to 38 with a median number (interquartile range) of 14 (10 to 29) participants per group (excluding dropouts; the details are provided in Table 1). Four of the 26 trials used a cross-over design $[15,19,36,40]$ and two trials used a within-participant design where the unit of analysis was the limb, not the participant $[1,2]$. Four of the 26 trials included more than two groups [4, 5, 16, 31].

All 26 trials measured strength in either Newtons $(\mathrm{N})$, $\mathrm{Nm}$ or points from a manual muscle test (typically summed across multiple muscles). The Risk of Bias in the trials is provided in Figs. 2-4. In brief, 12 of the 26 trials were at high risk of bias on three or more items of the Cochrane Risk of Bias Tool. Bias was most commonly due to failure to conceal allocation, blind participants and personnel, and blind assessors. 
Table 1 Details of the included studies

A. Trials comparing an intervention to a sham or no intervention (see Fig. 2)

\begin{tabular}{|c|c|c|c|c|c|c|c|}
\hline Study & Comparison & Dosage & Target muscle & RCT Design & $N$ & Participants & Strength measure \\
\hline Bye et al. [1] & $\begin{array}{l}\text { Exp: Resistance training }+ \\
\text { usual care } \\
\text { Cont: Usual care }\end{array}$ & $\begin{array}{l}-40 \text { max. } \\
\text { contractions } \\
\cdot 3 \text { times per wk } \\
\cdot 12 \text { wks }\end{array}$ & $\begin{array}{l}\text { One of: } \\
\text { - elbow flexors } \\
\text { - elbow extensors } \\
\text { - knee flexors } \\
\text { - knee extensors } \\
\text { (>grade } \\
3 \text { strength) }\end{array}$ & $\begin{array}{l}\text { Within- } \\
\text { participant }\end{array}$ & $30 / 30$ & $\begin{array}{l}\cdot<1 \text { year } \\
\cdot \text { C1-L3 } \\
\cdot \text { AIS A-D } \\
\text { - } 80 \% \text { male }\end{array}$ & Torque $(\mathrm{Nm})$ \\
\hline $\begin{array}{l}\text { Glinsky et al. } \\
{[22]}\end{array}$ & $\begin{array}{l}\text { Exp: Resistance training } \\
\text { Cont: No intervention }\end{array}$ & $\begin{array}{l}\text { - } 30 \text { max. } \\
\text { contractions } \\
\text { - } 3 \text { times per wk } \\
\text { - } 8 \mathrm{wks}\end{array}$ & $\begin{array}{l}\text { One of: } \\
\text { - wrist flexors } \\
\text { - wrist extensors }\end{array}$ & $\begin{array}{l}\text { Between- } \\
\text { participant }\end{array}$ & $31 / 32$ & $\begin{array}{l}\cdot>2 \text { months } \\
\cdot \text { C4-C7 } \\
\cdot \text { AIS A-D } \\
\cdot 87 \% \text { male }\end{array}$ & Torque $(\mathrm{Nm})$ \\
\hline $\begin{array}{l}\text { Beekhuizen } \\
\text { et al. [20] }\end{array}$ & $\begin{array}{l}\text { Exp: Somatosensory } \\
\text { stimulation }+ \text { massed } \\
\text { Practice } \\
\text { Cont: Massed practice }\end{array}$ & $\begin{array}{l}\cdot 2 \mathrm{~h} \\
\cdot 5 \text { times per wk } \\
\text { - } 3 \mathrm{wks}\end{array}$ & Hand muscles & $\begin{array}{l}\text { Between- } \\
\text { participant }\end{array}$ & $10 / 10$ & $\begin{array}{l}\text { - }>1 \text { year } \\
\text { - C5-C7 } \\
\text { - AIS C-D } \\
\text { - } 90 \% \text { male }\end{array}$ & Pinch Grip (N) \\
\hline $\begin{array}{l}\text { Beekhuizen } \\
\text { et al. [4] }\end{array}$ & $\begin{array}{l}\text { Exp: Massed practice }+ \\
\text { somatosensory stimulation } \\
\text { Cont: No intervention }\end{array}$ & $\begin{array}{l}\cdot 2 \mathrm{~h} \\
\cdot 5 \text { days per wk } \\
\cdot 3 \text { wks }\end{array}$ & Hand muscles & $\begin{array}{l}\text { Between- } \\
\text { participant }\end{array}$ & $12 / 12$ & $\begin{array}{l}\cdot>1 \text { year } \\
\cdot \text { C5-C7 } \\
\cdot \text { AIS C-D } \\
\cdot 83 \% \text { male }\end{array}$ & Pinch grip (Kg) \\
\hline $\begin{array}{l}\text { Harvey et al. } \\
{[24]}\end{array}$ & $\begin{array}{l}\text { Exp: Hand therapy with } \\
\text { Rejoyce }+ \text { FES }+15 \text { min } \\
\text { hand therapy } \\
\text { Cont: } 15 \text { mins hand therapy }\end{array}$ & $\begin{array}{l}\cdot 1 \mathrm{~h} \\
\cdot 5 \text { times per wk } \\
\cdot 8 \mathrm{wks}\end{array}$ & $\begin{array}{l}\text { Upper limb } \\
\text { muscles }\end{array}$ & $\begin{array}{l}\text { Between- } \\
\text { participant }\end{array}$ & $66 / 70$ & $\begin{array}{l}\cdot<6 \text { months } \\
\cdot \text { C } 1-\mathrm{C} 7 \\
\cdot \text { AIS A-D } \\
\cdot 87 \% \text { male }\end{array}$ & $\begin{array}{l}\text { Summed UL } \\
\text { strength of the } \\
\text { GRASSP (/50 } \\
\text { points) }\end{array}$ \\
\hline $\begin{array}{l}\text { Harvey et al. } \\
\text { [23] }\end{array}$ & $\begin{array}{l}\text { Exp: Resistance training }+ \\
\text { ES } \\
\text { Cont: No intervention }\end{array}$ & $\begin{array}{l}-120 \text { max. } \\
\text { contractions } \\
\text { - } 3 \text { times per wk } \\
\text { - } 8 \text { wks }\end{array}$ & $\begin{array}{l}\text { Quadriceps } \\
\text { muscles }\end{array}$ & $\begin{array}{l}\text { Between- } \\
\text { participant }\end{array}$ & $20 / 20$ & $\begin{array}{l}\cdot>6 \text { months } \\
\cdot \text { C5-L2 } \\
\cdot \text { AIS C-D } \\
\cdot 70 \% \text { male }\end{array}$ & Torque $(\mathrm{Nm})$ \\
\hline $\begin{array}{l}\text { Glinsky et al. } \\
{[2]}\end{array}$ & $\begin{array}{l}\text { Exp: Resistance training + } \\
\text { ES } \\
\text { Cont: Resistance training } \\
+ \text { Sham ES }\end{array}$ & $\begin{array}{l}\text { - } 60 \text { max. } \\
\text { contractions } \\
\text { - } 3 \text { times per wk } \\
\text { - } 8 \mathrm{wks}\end{array}$ & $\begin{array}{l}\text { One of: } \\
\text { - wrist flexors } \\
\text { - wrist extensors }\end{array}$ & $\begin{array}{l}\text { Within- } \\
\text { participant }\end{array}$ & $32 / 32$ & $\begin{array}{l}\cdot>2 \text { months } \\
\cdot \text { C } 4-\mathrm{C} 7 \\
\cdot \text { NP } \\
\cdot 88 \% \text { male }\end{array}$ & Torque $(\mathrm{Nm})$ \\
\hline Kim et al. [26] & $\begin{array}{l}\text { Exp: Arm cranking } \\
\text { exercise } \\
\text { Cont: No intervention }\end{array}$ & $\begin{array}{l}\cdot 1 \mathrm{~h} \\
\cdot 3 \text { times per wk } \\
\text { - } 6 \mathrm{wks}\end{array}$ & Elbow extensors & $\begin{array}{l}\text { Between- } \\
\text { participant }\end{array}$ & $15 / 16$ & $\begin{array}{l}\cdot>2 \text { year } \\
\text { - C5-T11 } \\
\text { - AIS A-B } \\
\text { - } 60 \% \text { male }\end{array}$ & Newton $(\mathrm{N})$ \\
\hline Klose et al. [27] & $\begin{array}{l}\text { Exp: } \text { Biofeedback }+ \\
\text { strength training }+ \text { ES } \\
\text { Cont: } \text { Strength training }+ \\
\text { ES }\end{array}$ & $\begin{array}{l}\cdot 30 \text { mins } \\
\cdot 3 \text { times per wk } \\
\cdot 12 \mathrm{wks}\end{array}$ & $\begin{array}{l}\text { Upper limb } \\
\text { muscles }\end{array}$ & $\begin{array}{l}\text { Between- } \\
\text { participant }\end{array}$ & $28 / 31$ & $\begin{array}{l}\cdot>1 \text { year } \\
\cdot \text { C5-C7 } \\
\cdot \text { NP } \\
\cdot 86 \% \text { male }\end{array}$ & $\begin{array}{l}\text { Summed MMT of } \\
8 \text { UL muscles ( } / 40 \\
\text { points) }\end{array}$ \\
\hline Jones et al. [25] & $\begin{array}{l}\text { Exp: A package of PT } \\
\text { interventions } \\
\text { Cont: No intervention }\end{array}$ & $\begin{array}{l}\cdot 3 \mathrm{~h} \\
\cdot 3 \text { times per wk } \\
\cdot 24 \mathrm{wks}\end{array}$ & $\begin{array}{l}\text { Lower limb } \\
\text { muscles }\end{array}$ & $\begin{array}{l}\text { Between- } \\
\text { participant }\end{array}$ & $41 / 48$ & $\begin{array}{l}\cdot>1 \text { year } \\
\cdot \text { C2-T10 } \\
\text { - AIS C-D } \\
\text { - } 77 \% \text { male }\end{array}$ & LEMS (/50 points) \\
\hline
\end{tabular}

B. Trials comparing one type of locomotor intervention to another type of locomotor or physiotherapy intervention (see Fig. 3)

\begin{tabular}{|c|c|c|c|c|c|c|c|}
\hline Alexeeva et al. [31] & $\begin{array}{l}\text { Exp: BWSTT } \\
\text { Cont: Overground gait } \\
\text { training and usual care }\end{array}$ & $\begin{array}{l}-1 \mathrm{~h} \\
\text { - } 3 \mathrm{times} \\
\text { per wk } \\
\text { - } 13 \mathrm{wks}\end{array}$ & $\begin{array}{l}\text { Upper and } \\
\text { lower limb } \\
\text { muscles }\end{array}$ & $\begin{array}{l}\text { Between- } \\
\text { participant }\end{array}$ & $21 / 21$ & $\begin{array}{l}\cdot>1 \text { year } \\
\cdot \text { C4-T10 } \\
\text { - AIS C-D } \\
\text { - } 86 \% \text { male }\end{array}$ & $\begin{array}{l}\text { UEMS and } \\
\text { LEMS }(/ 100 \\
\text { points })\end{array}$ \\
\hline Field-Fote et al. [5] & $\begin{array}{l}\text { Exp: BWSTT + ES } \\
\text { Cont: } \text { Overground gait } \\
\text { training + ES }\end{array}$ & $\begin{array}{l}\text { - } 30 \mathrm{mins} \\
\text { - } 5 \text { times } \\
\text { per wk } \\
\text { - } 12 \mathrm{wks}\end{array}$ & $\begin{array}{l}\text { Right lower } \\
\text { limb muscles }\end{array}$ & $\begin{array}{l}\text { Between- } \\
\text { participant }\end{array}$ & $33 / 40$ & $\begin{array}{l}\cdot>1 \text { year } \\
\cdot \text { above T10 } \\
\text { - AIS C-D } \\
\text { - } 75 \% \text { male }\end{array}$ & $\begin{array}{l}\text { Right LEMS } \\
\text { (/25 points) }\end{array}$ \\
\hline
\end{tabular}


Table 1 (continued)

B. Trials comparing one type of locomotor intervention to another type of locomotor or physiotherapy intervention (see Fig. 3)

\begin{tabular}{|c|c|c|c|c|c|c|c|}
\hline $\begin{array}{l}\text { Alcobendas- Maestro } \\
\text { et al. [6] }\end{array}$ & $\begin{array}{l}\text { Exp: Robotic gait training } \\
\text { Cont: Overground gait } \\
\text { training }\end{array}$ & $\begin{array}{l}-1 \mathrm{~h} \\
-5 \mathrm{times} \\
\text { per wk } \\
-8 \mathrm{wks}\end{array}$ & $\begin{array}{l}\text { Lower limb } \\
\text { muscles }\end{array}$ & $\begin{array}{l}\text { Between- } \\
\text { participant }\end{array}$ & $75 / 80$ & $\begin{array}{l}\cdot<6 \text { months } \\
\cdot \text { C2-T12 } \\
\cdot \text { AIS C-D } \\
\cdot 63 \% \text { male }\end{array}$ & $\begin{array}{l}\text { LEMS (/50 } \\
\text { points) }\end{array}$ \\
\hline Hornby et al. [7] & $\begin{array}{l}\text { Exp: Robotic assisted } \\
\text { BWSTT } \\
\text { Cont: Overground gait } \\
\text { training }\end{array}$ & $\begin{array}{l}\text { - } 30 \mathrm{mins} \\
\text { - } 3 \text { times } \\
\text { per wk } \\
\text { - } 8 \mathrm{wks}\end{array}$ & $\begin{array}{l}\text { Lower limb } \\
\text { muscles }\end{array}$ & $\begin{array}{l}\text { Between- } \\
\text { participant }\end{array}$ & 20/NP & $\begin{array}{l}-<6 \text { months } \\
\cdot \text { above T10 } \\
\text { - AIS B-D } \\
\cdot \text { NP }\end{array}$ & $\begin{array}{l}\text { LEMS (/50 } \\
\text { points) }\end{array}$ \\
\hline Shin et al. [44] & $\begin{array}{l}\text { Exp: Robotic gait training } \\
\text { Cont: Overground gait } \\
\text { training }\end{array}$ & $\begin{array}{l}\text { - } 40 \text { mins } \\
\text { - } 3 \text { times } \\
\text { per wk } \\
\text { - } 4 \text { wks }\end{array}$ & $\begin{array}{l}\text { Lower limb } \\
\text { muscles }\end{array}$ & $\begin{array}{l}\text { Between- } \\
\text { participant }\end{array}$ & $53 / 60$ & $\begin{array}{l}-<6 \text { months } \\
\text { - above L1 } \\
\text { - AIS D } \\
\text { - } 64 \% \text { male }\end{array}$ & $\begin{array}{l}\text { LEMS (/50 } \\
\text { points) }\end{array}$ \\
\hline Labruyere [19] & $\begin{array}{l}\text { Exp: Robotic gait training } \\
\text { Cont: Strength training }\end{array}$ & $\begin{array}{l}\text { - } 45 \text { mins } \\
\text { - } 4 \text { times } \\
\text { per week } \\
\text { - } 4 \text { wks }\end{array}$ & $\begin{array}{l}\text { Lower limb } \\
\text { muscles }\end{array}$ & Cross-over & $9 / 9$ & $\begin{array}{l}\cdot>1 \text { year } \\
\cdot \text { C4-T11 } \\
\text { - AIS C-D } \\
\text { - } 56 \% \text { male }\end{array}$ & $\begin{array}{l}\text { LEMS (/50 } \\
\text { points) }\end{array}$ \\
\hline Wu et al. [46] & $\begin{array}{l}\text { Exp: } \text { Swing-assisted } \\
\text { robotic gait training } \\
\text { Cont: Swing-resisted } \\
\text { robotic gait training }\end{array}$ & $\begin{array}{l}\text { - } 45 \text { mins } \\
\text { - } 3 \text { times } \\
\text { per wk } \\
\text { - } 6 \text { wks }\end{array}$ & $\begin{array}{l}\text { Lower limb } \\
\text { muscles }\end{array}$ & $\begin{array}{l}\text { Between- } \\
\text { participant }\end{array}$ & $12 / 14$ & $\begin{array}{l}\cdot>1 \text { year } \\
\text { - C2-T10 } \\
\text { - AIS CD } \\
\text { - } 75 \% \text { male }\end{array}$ & $\begin{array}{l}\text { LEMS (/50 } \\
\text { points) }\end{array}$ \\
\hline Brazg et al. [15] & $\begin{array}{l}\text { Exp: High-intensity } \\
\text { BWSTT + overground } \\
\text { gait training } \\
\text { Cont }: \text { Low-intensity } \\
\text { BWSTT + overground } \\
\text { gait training }\end{array}$ & $\begin{array}{l}\bullet 1 \mathrm{~h} \\
\bullet 3-5 \\
\text { times per } \\
\text { wk } \\
\cdot 4-6 \mathrm{wks}\end{array}$ & $\begin{array}{l}\text { Lower limb } \\
\text { muscles }\end{array}$ & Cross-over & $15 / 17$ & $\begin{array}{l}\text { - }>1 \text { year } \\
\text { - C1-T10 } \\
\text { - AIS C-D } \\
\text { - } 73 \% \text { male }\end{array}$ & $\begin{array}{l}\text { LEMS (/50 } \\
\text { points) }\end{array}$ \\
\hline $\begin{array}{l}\text { Senthilvelkumar et al. } \\
\text { [43] }\end{array}$ & $\begin{array}{l}\text { Exp: BWS overground gait } \\
\text { training } \\
\text { Cont: BWSTT }\end{array}$ & $\begin{array}{l}\cdot 30 \mathrm{mins} \\
\text { - } 5 \text { times } \\
\text { per wk } \\
\text { - } 8 \mathrm{wks}\end{array}$ & $\begin{array}{l}\text { Lower limb } \\
\text { muscles }\end{array}$ & $\begin{array}{l}\text { Between- } \\
\text { participant }\end{array}$ & $14 / 16$ & $\begin{array}{l}-<2 \text { years } \\
\cdot \text { C5-C8 } \\
\text { - AIS C } \\
\text { - } 79 \% \text { male }\end{array}$ & $\begin{array}{l}\text { LEMS (/50 } \\
\text { points) }\end{array}$ \\
\hline Gorman et al. [33] & $\begin{array}{l}\text { Exp: Robotic gait training } \\
\text { Cont: Stretching }\end{array}$ & $\begin{array}{l}-20-45 \\
\text { mins } \\
\text { - } 3 \text { times } \\
\text { per wk } \\
\text { - } 12 \mathrm{wks}\end{array}$ & $\begin{array}{l}\text { Lower limb } \\
\text { muscles }\end{array}$ & $\begin{array}{l}\text { Between- } \\
\text { participant }\end{array}$ & $18 / 18$ & $\begin{array}{l}\cdot>1 \text { year } \\
\cdot \text { C4-L2 } \\
\cdot \text { AIS C-D } \\
\cdot \text { NP }\end{array}$ & $\begin{array}{l}\text { LEMS (/50 } \\
\text { points) }\end{array}$ \\
\hline
\end{tabular}

C. Trials comparing one type of intervention to another type of intervention (see Fig. 4)

\begin{tabular}{|c|c|c|c|c|c|c|c|}
\hline Study & Comparison & Dosage & Target muscle & Design & $N$ & Participants & $\begin{array}{l}\text { Strength } \\
\text { measure }\end{array}$ \\
\hline $\begin{array}{l}\text { Gomes-Osman et al. } \\
{[16]}\end{array}$ & $\begin{array}{l}\text { Exp: Functional task } \\
\text { training + somatosensory } \\
\text { stimulation } \\
\text { Cont: Conventional } \\
\text { exercise training }\end{array}$ & $\begin{array}{l}\cdot 2 \mathrm{~h} \\
\cdot 5 \text { times } \\
\text { per wk } \\
\text { - } 4 \text { wks }\end{array}$ & Hand muscles & $\begin{array}{l}\text { Between- } \\
\text { participant }\end{array}$ & $24 / \mathrm{NP}$ & $\begin{array}{l}\cdot>1 \text { year } \\
\cdot \text { C4-C8 } \\
\cdot \text { AIS A-D } \\
\cdot \text { NP }\end{array}$ & Power grip $(\mathrm{N})$ \\
\hline Hoffman et al. [35] & $\begin{array}{l}\text { Exp: Bimanual hand } \\
\text { training + somatosensory } \\
\text { stimulation } \\
\text { Cont: Unimanual hand } \\
\text { training + somatosensory } \\
\text { stimulation }\end{array}$ & $\begin{array}{l}\text { - } 3 \mathrm{~h} \\
\text { - } 5 \text { times } \\
\text { per wk } \\
\text { - } 3 \text { wks }\end{array}$ & Hand muscles & $\begin{array}{l}\text { Between- } \\
\text { participant }\end{array}$ & $11 / 13$ & $\begin{array}{l}\cdot>1 \text { year } \\
\cdot \text { C3-C7 } \\
\cdot \text { AIS B-D } \\
\cdot 77 \% \text { male }\end{array}$ & Pinch grip (N) \\
\hline Kapadia et al. [37] & $\begin{array}{l}\text { Exp: FES + strengthening } \\
\text { Cont: }: \text { ES + strengthening } \\
+ \text { hand therapy }\end{array}$ & $\begin{array}{l}-1 \mathrm{~h} \\
\text { - } 3 \text { times } \\
\text { per wk } \\
\text { - } 13 \mathrm{wks}\end{array}$ & Hand muscles & $\begin{array}{l}\text { Between- } \\
\text { participant }\end{array}$ & $8 / 8$ & $\begin{array}{l}\cdot<2 \text { years } \\
\cdot \text { C4-C7 } \\
\cdot \text { AIS B-D } \\
\cdot \text { NP }\end{array}$ & $\begin{array}{l}\text { Palmar grasp } \\
(\mathrm{Nm})\end{array}$ \\
\hline
\end{tabular}


Table 1 (continued)

C. Trials comparing one type of intervention to another type of intervention (see Fig. 4)

\begin{tabular}{|c|c|c|c|c|c|c|c|}
\hline Study & Comparison & Dosage & Target muscle & Design & $N$ & Participants & $\begin{array}{l}\text { Strength } \\
\text { measure }\end{array}$ \\
\hline Kowalczewski et al. [40] & $\begin{array}{l}\text { Exp: FES + hand therapy } \\
\text { with ReJoyce } \\
\text { Cont: } \mathrm{ES}+\text { hand therapy } \\
\text { with computer + strength } \\
\text { training }\end{array}$ & $\begin{array}{l}\text { - } 1 \mathrm{~h} \\
\text { - } 5 \text { times } \\
\text { per wk } \\
\text { - } 6 \text { wks }\end{array}$ & Hand muscles & Cross-over & $13 / 13$ & $\begin{array}{l}\cdot>9 \text { mnths } \\
\cdot \text { C5-C7 } \\
\cdot \text { NP } \\
\cdot 54 \% \text { male }\end{array}$ & Pinch grip (N) \\
\hline Galea et al. [32] & $\begin{array}{l}\text { Exp: FES cycling } \\
\text { Cont: } \text { Passive cycling }\end{array}$ & $\begin{array}{l}\text { - } 1 \mathrm{~h} \\
\text { - } 4 \text { times } \\
\text { per wk } \\
\text { - } 12 \mathrm{wks}\end{array}$ & $\begin{array}{l}\text { Lower limb } \\
\text { muscles }\end{array}$ & $\begin{array}{l}\text { Between- } \\
\text { participant }\end{array}$ & $21 / 24$ & $\begin{array}{l}\cdot<4 \text { wks } \\
\text { - above T12 } \\
\text { - AIS A-C } \\
\text { - } 96 \% \text { male }\end{array}$ & $\begin{array}{l}\text { LEMS (/50 } \\
\text { points) }\end{array}$ \\
\hline Jayaraman et al. [36] & $\begin{array}{l}\text { Exp: Maximal intensity } \\
\text { resistance training } \\
\text { Cont: Conventional } \\
\text { resistance training }\end{array}$ & $\begin{array}{l}\text { - } 30 \text { max. } \\
\text { contrac- } \\
\text { tions } \\
\text { - } 3 \text { times } \\
\text { per wk } \\
\text { - } 4 \text { wks }\end{array}$ & $\begin{array}{l}\text { Lower limb } \\
\text { muscles }\end{array}$ & Cross-over & $5 / 5$ & $\begin{array}{l}\cdot>4 \text { years } \\
\cdot \text { C2-T7 } \\
\cdot \text { AIS C-D } \\
\cdot 100 \% \text { male }\end{array}$ & $\begin{array}{l}\text { LEMS (/50 } \\
\text { points) }\end{array}$ \\
\hline
\end{tabular}

Comparison refers to the two groups included in this review. The experimental group is as defined for this review and as presented in the forest plots Dosage refers to the amount of therapy provided to the experimental group. Control groups that received therapy often received the same dosage but not always (these details are not provided)

$N$ refers to the number of participants who contributed to the analysis and the number of participants randomised. For the three studies with more than 2 groups, only the number of participants in the two groups included in the analyses are reported. For within-participant studies, the number refers to the participants (not the number of limbs)

Participants refers to time since injury, neurological or motor level (the distinction was often not clarified in the papers), AIS classification and sex $R C T$ randomised controlled trial, $N$ number of participants, Exp experimental, Cont control, max. maximal, wks weeks, mnths months, AIS American Spinal Injuries Association Impairment Scale, FES functional electrical stimulation, ES electrical stimulation, $h$ hours, mins minutes, $B W S T T$ body-weight supported treadmill training, BWS body-weight supported, Nm Newton metres, $N$ Newton, $K g$ kilogram, LEMS Lower Extremity Motor Scores, UEMS Upper Extremity Motor Scores, NP not provided, UL upper limb, GRASSP Graded and Redefined Assessment of Strength, Sensibility and Prehension, MMT manual muscle test

\section{Trials comparing a PT intervention to a sham or no intervention}

Ten trials compared a PT intervention to a sham or no intervention [1, 2, 4, 20, 22-27]. The results of the ten trials are split across two figures (see Fig. 2a and Fig. 2b).

Figure 2a includes five trials that examined the effect of two interventions: resistance training (two trials) and hand therapy (three trials). The results of these five trials were pooled in two meta-analyses (fixed effects) with results expressed as SMD. The meta-analyses investigating the effect of resistance training showed a statistically significant between-group difference $(\mathrm{SMD}=0.64 ; 95 \% \mathrm{CI}, 0.22-1.07 ; p=0.003)$.

Figure $2 \mathrm{~b}$ includes the results of the remaining five trials which compared one intervention to a sham or no intervention. These trials examined the effect of five different interventions: resistance training plus electrical stimulation, electrical stimulation, arm cranking exercise, biofeedback, and a package of PT interventions including locomotor training (see Table 1A). The results of the five trials were not pooled because of clinical heterogeneity, but instead are presented individually (see Fig. 2b). The results are expressed as MD with different units for each comparison. Two of the five trials indicated a statistically significant between-group difference: resistance training combined with electrical stimulation $(\mathrm{MD}=14 \mathrm{Nm} ; 95 \% \mathrm{CI}, 1-27 ; p=0.03)$ and a package of PT interventions including resistance training combined with locomotor training ( $\mathrm{MD}=4.8 / 50$ points on the LEMS; 95\% CI 1.9-7.7; $p=0.01$ ).

\section{Trials comparing any two PT interventions to each other}

Sixteen trials compared one type of PT intervention with another type [5-7, 15, 16, 19, 31-33, 35-37, 40, 43, 44, 46]. These 16 trials are split across two figures (see Figs. 3 and 4).

Figure 3 includes 10 trials that compared one type of gait training with another type of gait training (or another type of PT intervention): BWSTT versus overground gait training (two trials), robotic gait training versus overground gait training (three trials), robotic gait training versus strength training (one trial), swing-assisted robotic gait training versus swing-resisted robotic gait training (one trial), high-intensity BWSTT and overground gait training versus low-intensity BWSTT and 

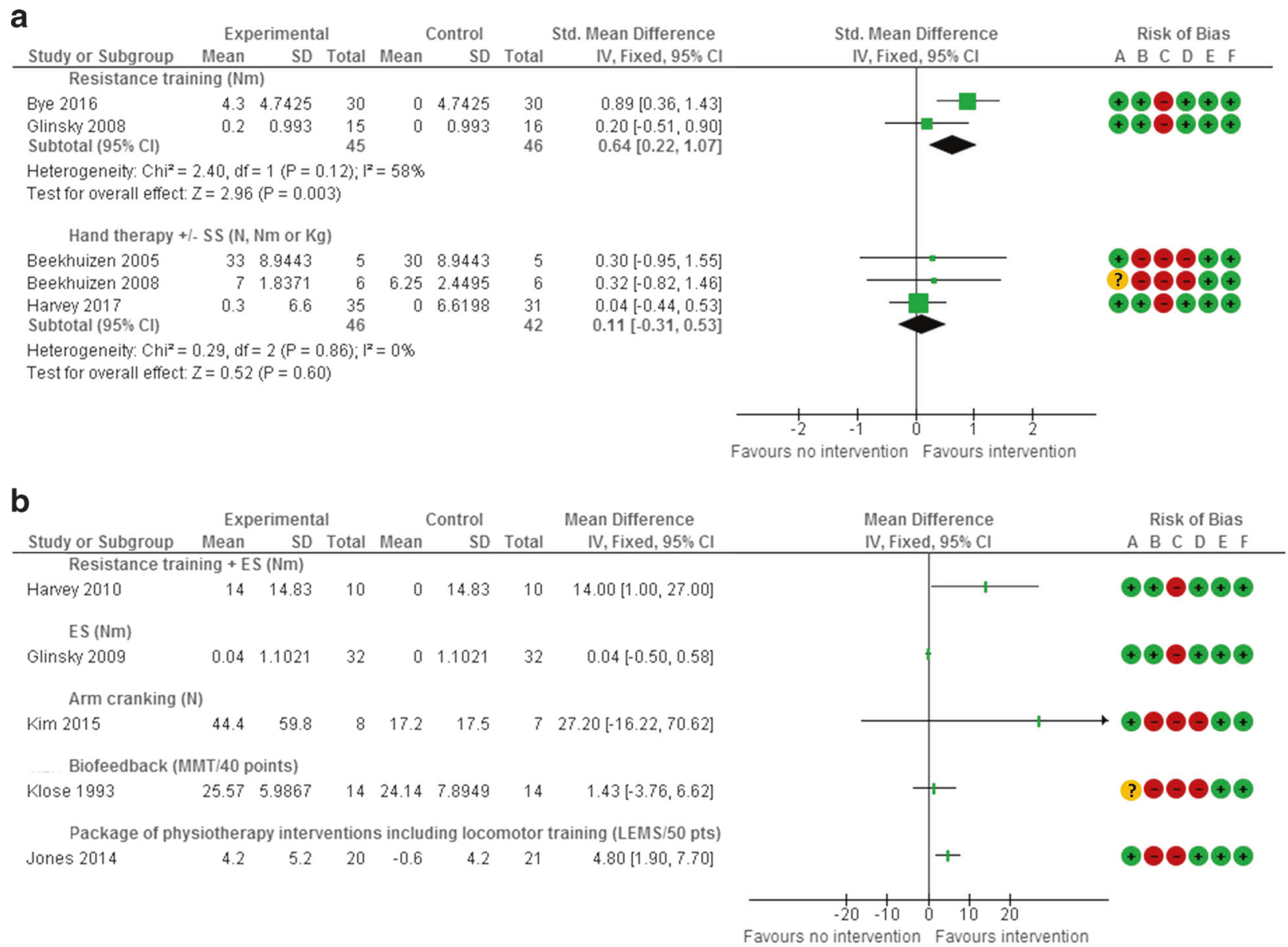

Fig. 2 Forest plot of trials comparing an intervention to no intervention or sham intervention expressed as standardised mean difference or mean difference. The figure includes trials which provided the same intervention to both groups but an additional intervention to the experimental group. Meta-analyses were only performed in the comparisons in (a). The results of each trial in (b) should be interpreted independently. The data from Jones 2014 are change data (no post data were provided), and the data from Bye 2016, Glinsky 2008, Glinsky 2009, Harvey 2010 and Harvey 2017 are back converted from mean between-group differences $(95 \% \mathrm{CI})$. All other data are post data. The risk of bias on each item of the Cochrane Risk of Bias Tool for the

overground gait training (one trial), body-weight supported overground gait training versus BWSTT (one trial), and robotic gait training versus stretch (one trial). All trials measured lower limb strength with a manual muscle test expressed as LEMS for both legs (/50 points), LEMS for one leg (/25 points) or a composite score of 10 muscles in each leg (/100 pts). For the purpose of analyses, all results were converted to a 50-point scale and presented as MD in Fig. 3. The results of the trials examining two comparisons (BWSTT versus overground gait training; robotic gait training versus overground gait training) were pooled in two meta-analyses (fixed effects) with results expressed as MD. One of the two meta-analyses comparing robotic gait training with overground gait training showed a statistically significant between-group difference $(\mathrm{MD}=3.1 / 50$ trials are indicated by the coloured circles, where green reflects low risk of bias, yellow reflects unclear risk of bias and red reflects high risk of bias. The Risk of Bias refers to A: Random sequence generation (selection bias); B: Concealment of allocation (selection bias); C: Blinding of participants and personnel (performance bias); D: Blinding of assessors (detection bias); E: Incomplete outcome data (attrition bias); F: Selective reporting (reporting bias). SS somatosensory stimulation, ES electrical stimulation, MMT manual muscle test, LEMS lower extremity motor score, $\mathrm{N}$ newton, $\mathrm{Nm}$ Newton/metre, and $\mathrm{Kg}$ kilogram

points on the LEMS; 95\% CI, $1.3-5.0 ; p=0.0008)$. The results of the other five trials were not included in metaanalyses and are presented individually. None of these trials showed a statistically significant between-group difference.

Figure 4 has the results of the remaining six trials which compared one type of non-gait related therapy with another type of non-gait related therapy. These trials examined three comparisons: one combination of hand therapies versus another combination of hand therapies (four trials), FES cycling versus passive leg cycling (one trial) and maximal intensity resistance training versus conventional resistance training (one trial). The results of the six trials were not pooled because the comparisons were not similar. Instead the results of each trial are 


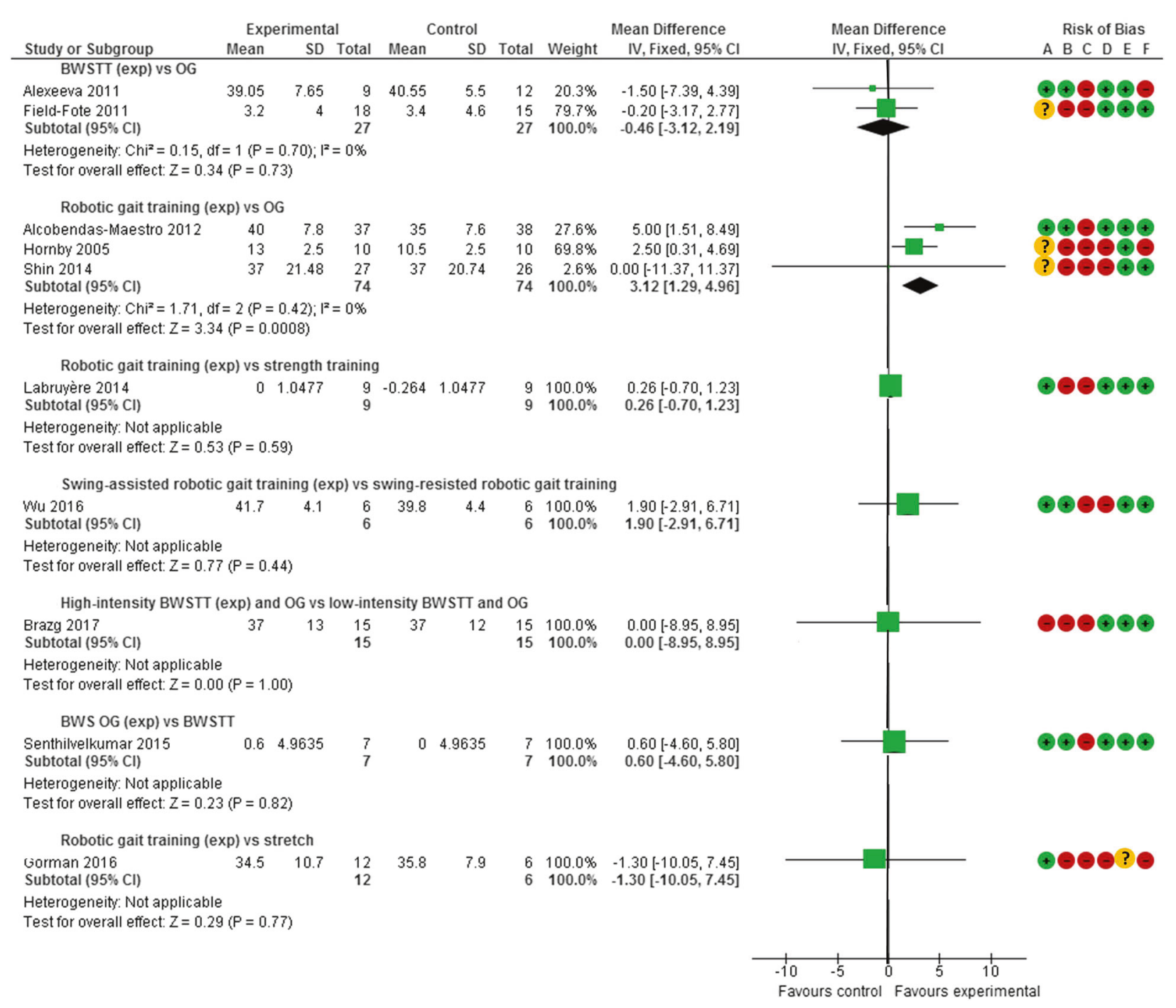

Fig. 3 Forest plot of trials comparing one type of locomotor intervention to another type of locomotor or physiotherapy intervention expressed as mean difference. The data from Hornby 2005 and FieldFote 2011 are change data, and the data from Labruyere 2014 are back converted from mean between-group differences $(95 \% \mathrm{CI})$. The data from Senthilvelkumar 2015 was back converted from mean betweengroup difference data $(95 \% \mathrm{CI})$ calculated using individual data attained from the authors (the authors acknowledge in a personal communication that the mean $(95 \% \mathrm{CI})$ data reported in their paper are

presented individually and expressed as MD with different units for each comparison (see Fig. 4). None of these trials showed a statistically significant between-group difference.

\section{Trials that did not provide useable data}

Of the 12 trials that did not provide useable data, seven did not report any results for between-group statistical comparisons [30, 34, 39, 41, 42, 45, 47]; three reported a nonsignificant between-group difference [3,21,38]; and one incorrect). All other data are post data. The data from Field-Fote 2011 were converted from a 25-point scale (composite measure of lower limb manual muscle tests for right leg) to a 50-point scale; and the data from Alexeeva 2011 were converted from a 100-point scale (composite measure of lower limb manual muscle tests from 10 lower limb muscles) to a 50-point scale. The definition of the experimental group is based on the intervention that is most effective. The risk of bias: see legend for Fig. 2. BWSTT body-weight supported treadmill training, $O G$ over-ground gait training

stated the difference between the groups was significant but the reported $p$ value indicated otherwise [29]. One trial reported a between-group statistical difference for neuromuscular stimulated-assisted arm cycling versus arm cycling alone. The outcome for this trial was the number of triceps muscles that improved by more than one grade on the 6-point manual muscle test. However, there was a unit of analysis problem because both arms of some, but not all, participants contributed to the analysis even though participants, not arms, were randomised [28] (see the Supplementary File 1 for details). 


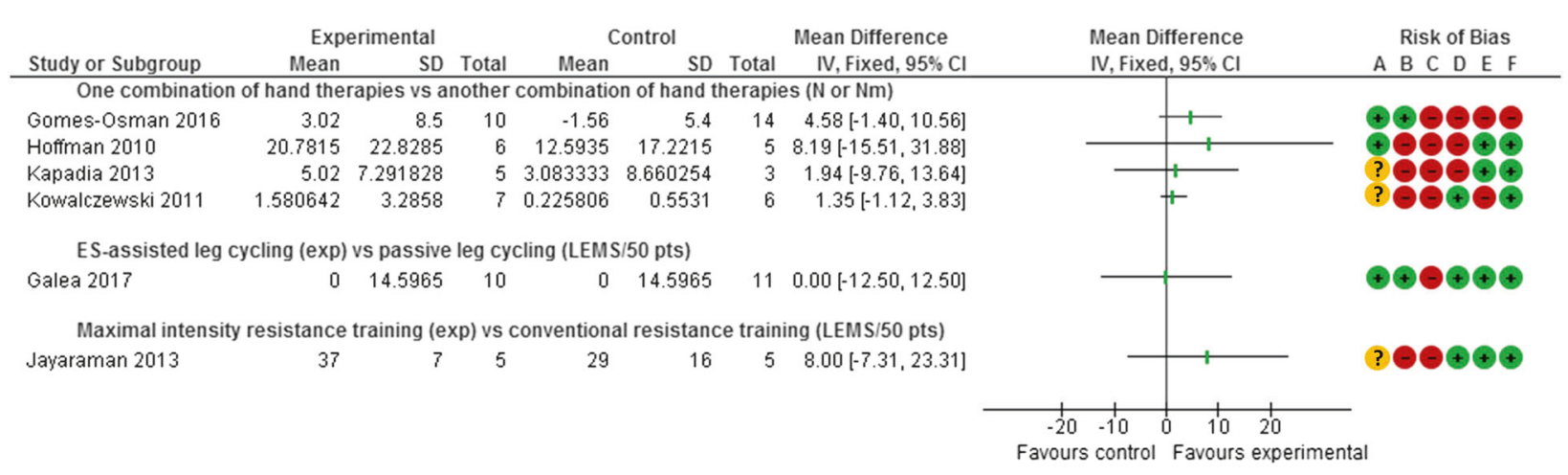

Fig. 4 Forest plot of trials comparing one type of non-gait related therapy with another type of non-gait related therapy expressed as mean difference. Meta-analyses were not performed in any of the comparisons including the comparison of hand therapies (for this comparison there was a mix of change and post data - see text for details). The data from Gomes-Osman 2016 and Kowalczewski 2011 are change data and the other data are post data. The data from Galea

\section{Discussion}

This systematic review is important because strength is central to the independence, mobility and quality of life of people with SCI. The strength of muscles directly affected by SCI is particularly important because this is often used as a measure of recovery [8-10]. The main finding from this review is that there is surprisingly little evidence to support the widely held belief that PT increases the strength of muscles directly affected by SCI. We were only able to find a statistically significant between-group difference for four comparisons, namely, resistance training versus no intervention; resistance training combined with electrical stimulation versus no intervention; a package of PT interventions including locomotor training versus no intervention; and robotic gait training versus overground gait training. However, the results from these four comparisons were not compelling because of the small numbers of trials and imprecise estimates. Of course, our failure to find more convincing evidence that PT interventions increase strength is not evidence that PT interventions are not effective. It does however suggest that there is considerable uncertainty around this topic.

Two types of comparisons were included in this review. The first included trials that compared any PT treatment with sham or no intervention. This comparison alone answers the question about the effectiveness of PT interventions. For this comparison we were only able to find evidence to support resistance training (two trials), resistance training combined with electrical stimulation (one trial) and a package of PT interventions including locomotor training (one trial). Interestingly, the package of PT interventions also included "resistance training" and "strengthening exercises" (pg 2246.e1) along with locomotor training, making it impossible to determine the
2017 was derived from a median (95\% CI) between-group difference calculated and provided by the authors using a boot strapped median regression model. The definition of the experimental group is based on the more effective intervention. The risk of bias: see legend for Fig. 2. LEMS Lower Extremity Motor Scores, $N$ newton, $N m$ Newton/ metre

relative effectiveness of resistance and locomotor training for increasing strength [25]. However, taken together, the findings of these four trials point to the importance of resistance training alone or in combination with other interventions. This finding aligns with what is known about the role of resistance training for increasing strength in ablebodied individuals with neurologically-intact muscles [48].

The second comparison included trials that compared any two types of PT interventions to each other. This comparison provides no insights into the effectiveness of interventions per se. It only indicates whether one treatment is, or is not, superior to another. We only found a statistically significant between-group difference for strength (in this case, on the LEMS) from robotic gait training versus overground gait training. There was no other evidence that one type of intervention was superior to another. This may indicate that all interventions are equally effective or equally ineffective; it is not known without a control group.

We excluded trials investigating the effects of different PT interventions on upper limb muscles if $20 \%$ or more of participants had paraplegia because it cannot be assumed that neurologically-weak muscles respond in the same way to interventions as muscles directly affected by SCI. We believe that the distinction between the two types of muscles in people with SCI may be important and results should not be pooled within or across trials.

There are limitations to this review. For example, we did not include trials that were not published in English. This is a common limitation of systematic reviews that do not include bilingual authors. There is also the possibility that we introduced bias into our review because two of the authors are also authors on some of the included trials.

Our forest plots and meta-analyses did not include the results of the 12 trials which stated that they measured 
strength but either did not report any strength data [38, 39, $42,47]$ or did not provide sufficient data for inclusion in the forest plots [3, 21, 28-30, 34, 41, 45]. We also did not include trials which may have measured strength but did not state that strength was measured. Similarly, there may have been trials which were conducted but never reported. These are all potential sources of publication bias tending to overstate treatment effectiveness because authors are less likely to publish the results of negative trials than positive trials, and because authors commonly omit the details of outcomes which are either negatively affected by the intervention or not changed by the intervention. It is not clear how big these types of publication biases are within the SCI literature. However, they could be minimised by compulsory prospective trial registration. We also did not extract data for all possible comparisons in studies with more than two groups. Instead, we prioritised comparisons that were most likely to demonstrate between-group differences. For this reason our results may be overly optimistic.

In all, the results of this systematic review raise important questions about the effectiveness of PT interventions for increasing strength in muscles directly affected by SCI. The results of a small number of trials indicate that resistance training is effective yet most trials in the review did not show a convincing treatment effect. The results should not be interpreted as evidence that PT interventions are not effective. They should however be interpreted as evidence that we need to be far more cautious before just assuming that PT interventions increase the strength of muscles directly affected by SCI.

\section{Data archiving}

The authors will consider all reasonable requests for the data upon which this systematic review are based.

Acknowledgements LAH's position is supported by Icare and SIRA, NSW, Australia.

Funding This systematic review was in part funded by Icare, NSW, Australia.

Author contributions All authors conceived the review, contributed to culling papers, extracted data, rated trials for bias, interpreted results and wrote up the final paper.

\section{Compliance with ethical standards}

Conflict of interest The authors declare that they have no conflict of interest.

Publisher's note: Springer Nature remains neutral with regard to jurisdictional claims in published maps and institutional affiliations.

\section{References}

1. Bye EA, Harvey LA, Gambhir A, Kataria C, Glinsky JV, Bowden JL, et al. Strength training for partially paralysed muscles in people with recent spinal cord injury: a within-participant randomised controlled trial. Spinal Cord. 2017;55:460-65.

2. Glinsky J, Harvey L, van Es P, Chee S, Gandevia SC. The addition of electrical stimulation to progressive resistance training does not enhance the wrist strength of people with tetraplegia: a randomized controlled trial. Clin Rehabil. 2009;23:696-704.

3. Popovic MR, Thrasher TA, Adams ME, Takes V, Zivanovic V, Tonack MI. Functional electrical therapy: retraining grasping in spinal cord injury. Spinal Cord. 2006;44:143-51.

4. Beekhuizen KS, Field-Fote EC. Sensory stimulation augments the effects of massed practice training in persons with tetraplegia. Arch Phys Med Rehabil. 2008;89:602-8.

5. Field-Fote EC, Roach KE. Influence of a locomotor training approach on walking speed and distance in people with chronic spinal cord injury: a randomized clinical trial. Phys Ther. 2011;91:48-60.

6. Alcobendas-Maestro M, Esclarin-Ruz A, Casado-Lopez RM, Munoz-Gonzalez A, Perez-Mateos G, Gonzalez-Valdizan E, et al. Lokomat robotic-assisted versus overground training within 3 to 6 months of incomplete spinal cord lesion: randomized controlled trial. Neurorehabil Neural Repair. 2012;26:1058-63.

7. Hornby TG, Campbell DD, Zemon DH, Kahn JH. Clinical and quantitative evaluation of robotic-assisted treadmill walking to retrain ambulation after spinal cord injury. Top Spinal Cord Inj Rehabil. 2005;11:1-17.

8. Tuszynski MH, Steeves JD, Fawcett JW, Lammertse D, Kalichman M, Rask C, et al. Guidelines for the conduct of clinical trials for spinal cord injury as developed by the ICCP Panel: clinical trial inclusion/exclusion criteria and ethics. Spinal Cord. 2007;45:222-31.

9. Lammertse DP. Clinical trials in spinal cord injury: lessons learned on the path to translation. The 2011 International Spinal Cord Society Sir Ludwig Guttmann Lecture. Spinal Cord. 2013;51:2-9.

10. Steeves JD, Lammertse D, Curt A, Fawcett JW, Tuszynski MH, Ditunno JF, et al. Guidelines for the conduct of clinical trials for spinal cord injury (SCI) as developed by the ICCP panel: clinical trial outcome measures. Spinal Cord. 2007;45:206-21.

11. Harvey L, Lin CM, Glinsky J, De Wolf A. The effectiveness of physical interventions for people with spinal cord injuries: a systematic review. Spinal Cord. 2009;47:184-95.

12. Harvey LA, Glinsky JV, Bowden JL. The effectiveness of 22 commonly administered physiotherapy interventions for people with spinal cord injury: a systematic review. Spinal Cord. 2016;54:914-23.

13. Mehrholz J, Harvey LA, Thomas S, Elsner B. Is body-weightsupported treadmill training or robotic-assisted gait training superior to overground gait training and other forms of physiotherapy in people with spinal cord injury? A systematic review. Spinal Cord. 2017;55:722-9.

14. Dickersin K, Scherer R, Lefebvre C. Identifying relevant studies for systematic reviews. BMJ. 1994;309:1286-91.

15. Brazg G, Fahey M, Holleran CL, Connolly M, Woodward J, Hennessy PW, et al. Effects of training intensity on locomotor performance in individuals with chronic spinal cord injury: A randomized crossover study. Neurorehabil Neural Repair. 2017;31:944-54.

16. Gomes-Osman J, Tibbett JA, Poe BP, Field-Fote EC. Priming for improved hand strength in persons with chronic tetraplegia: a comparison of priming-augmented functional task practice, 
priming alone, and conventional exercise training. Front Neurol. 2016;7:242.

17. The Cochrane Collaboration. Cochrane Handbook for Systematic Reviews of Interventions Version 5. 1.0 [updated March 2011]. The Cochrane Collaboration, 2011. www.cochrane-handbook.org. 2011.

18. Review Manager (RevMan). The Nordic Cochrane Centre. Copenhagen: The Cochrane Collaboration; 2015.

19. Labruyère R, van Hedel HJ. Strength training versus robot-assisted gait training after incomplete spinal cord injury: A randomized pilot study in patients depending on walking assistance. J Neuroeng Rehabil. 2014;11:4. https://doi.org/10.1186/1743-0003-11-4.

20. Beekhuizen KS. New perspectives on improving upper extremity function after spinal cord injury. $J$ Neurol Phys Ther. 2005;29:157-62.

21. Dobkin B, Apple D, Barbeau H, Basso M, Behrman A, Deforge D, et al. Weight-supported treadmill vs over-ground training for walking after acute incomplete SCI. Neurology. 2006;66:484-493.

22. Glinsky J, Harvey L, Korten M, Drury C, Chee S, Gandevia SC. Short-term progressive resistance exercise may not be effective for increasing wrist strength in people with tetraplegia: a randomised controlled trial. Aust J Physiother. 2008;54:103-8.

23. Harvey LA, Fornusek C, Bowden JL, Pontifex N, Glinsky J, Middleton JW, et al. Electrical stimulation plus progressive resistance training for leg strength in spinal cord injury: a randomized controlled trial. Spinal Cord. 2010;48:570-5.

24. Harvey LA, Dunlop SA, Churilov L, Galea MP. Early intensive hand rehabilitation is not more effective than usual care plus oneto-one hand therapy in people with sub-acute spinal cord injury ('Hands On'): a randomised trial. J Physiother. 2017; 63:197-204.

25. Jones ML, Evans N, Tefertiller C, Backus D, Sweatman M, Tansey K. et al. Activity-Based Therapy for recovery of walking in individuals with chronic spinal cord injury: results from a randomized clinical trial. Arch Phys Med Rehabil. 2014; 95:2239-46.

26. Kim DI, Lee H, Lee BS, Kim J, Jeon JY. Effects of a six-week indoor hand-bike exercise program on health and fitness levels in people with spinal cord injury: a randomized controlled trial study. Arch Phys Med Rehabil. 2015: https://doi.org/10.1016/j. apmr.2015.1007.1010.

27. Klose KJ, Needham BM, Schmidt D, Broton JG, Green BA. An assessment of the contribution of electromyographic biofeedback as an adjunct therapy in the physical training of spinal cord injured persons. Arch Phys Med Rehabil. 1993;74:453-6.

28. Needham-Shropshire BM, Broton JG, Cameron TL, Klose KJ. Improved motor function in tetraplegics following neuromuscular stimulation-assisted arm ergometry. J Spinal Cord Med. 1997;20:49-55.

29. Popovic MR, Kapadia N, Zivanovic V, Furlan JC, Craven BC, McGillivray C. Functional electrical stimulation therapy of voluntary grasping versus only conventional rehabilitation for patients with subacute incomplete tetraplegia: a randomized clinical trial. Neurorehabil Neural Repair. 2011;25:433-42.

30. Varoqui D, Niu X, Mirbagheri MM. Ankle voluntary movement enhancement following robotic-assisted locomotor training in spinal cord injury. J Neuroeng Rehabil. 2014;11:46.

31. Alexeeva N, Sames C, Jacobs PL, Hobday L, Distasio MM, Mitchell SA, et al. Comparison of training methods to improve walking in persons with chronic spinal cord injury: a randomized clinical trial. J Spinal Cord Med. 2011;34:362-79.

32. Galea MP, Panisset MG, El-Ansary D, Dunlop SA, Marshall R, Clark JM, et al. SCIPA Switch-On: A Randomized Controlled Trial Investigating the Efficacy and Safety of Functional Electrical Stimulation-Assisted Cycling and Passive Cycling Initiated Early after Traumatic Spinal Cord Injury. Neurorehabil Neural Repair. 2017;31:540-51.

33. Gorman PH, Scott W, York H, Theyagaraj M, Price-Miller N, McQuaid J, et al. Robotically assisted treadmill exercise training for improving peak fitness in chronic motor incomplete spinal cord injury: a randomized controlled trial. J Spinal Cord Med. 2016;39:32-44.

34. Hartkopp A, Harridge SD, Mizuno M, Ratkevicius A, Quistorff B, Kjaer M, et al. Effect of training on contractile and metabolic properties of wrist extensors in spinal cord-injured individuals. Muscle Nerve. 2003;27:72-80.

35. Hoffman LR, Field-Fote EC. Functional and corticomotor changes in individuals with tetraplegia following unimanual or bimanual massed practice training with somatosensory stimulation: a pilot study. J Neurol Phys Ther. 2010;34:193-201.

36. Jayaraman A, Thompson CK, Rymer WZ, Hornby TG. Short-term maximal-intensity resistance training increases volitional function and strength in chronic incomplete spinal cord injury: a pilot study. J Neurol Phys Ther. 2013;37:112-7.

37. Kapadia N, Zivanovic V, Popovic MR. Restoring voluntary grasping function in individuals with incomplete chronic spinal cord injury: pilot study. Top Spinal Cord Inj Rehabil. 2013;19:279-87.

38. Klose KJ, Schmidt DL, Needham BM, Brucker BS, Green BA, Ayyar DR. Rehabilitation therapy for patients with long-term spinal cord injuries. Arch Phys Med Rehabil. 1990;71:659-62.

39. Kohlmeyer KM, Hill JP, Yarkony GM, Jaeger RJ. Electrical stimulation and biofeedback effect on recovery of tenodesis grasp: a controlled study. Arch Phys Med Rehabil. 1996;77:701-6.

40. Kowalczewski J, Chong SL, Galea M, Prochazka A. In-home telerehabilitation improves tetraplegic hand function. Neurorehabil Neural Repair. 2011;25:412-22.

41. Piira A, Lannem AM, Sorensen M, Glott T, Knutsen R, Gjesdal $\mathrm{N}$, et al. Effects of locomotor training in subjects with incomplete sci-a randomized controlled trial. Arch Phys Med Rehabil. 2017;98:e60-1.

42. Postans NJ, Hasler JP, Granat MH, Maxwell DJ. Functional electrical stimulation to augment partial weight-bearing supported treadmill training for patients with acute incomplete spinal cord injury: a pilot study. Arch Phys Med Rehabil. 2004;85:604-10.

43. Senthilvelkumar T, Magimairaj H, Fletcher J, Tharion G, George $\mathrm{J}$. Comparison of body weight-supported treadmill training versus body weight-supported overground training in people with incomplete tetraplegia: a pilot randomized trial. Clin Rehabil. 2015;29:42-9.

44. Shin JC, Kim JY, Park HK, Kim NY. Effect of robotic-assisted gait training in patients with incomplete spinal cord injury. Ann Rehabil Med. 2014;38:719-25.

45. Wu M, Landry JM, Schmit BD, Hornby TG, Yen SC. Robotic resistance treadmill training improves locomotor function in human spinal cord injury: a pilot study. Arch Phys Med Rehabil. 2012;93:782-9.

46. Wu M, Landry JM, Kim J, Schmit BD, Yen SC, McDonald J, et al. Repeat exposure to leg swing perturbations during treadmill training induces long-term tetention of increased step length in human SCI: A pilot randomized controlled study. Am J Phys Med Rehabil. 2016;95:911-20.

47. Yang JF, Musselman KE, Livingstone D, Brunton K, Hendricks $\mathrm{G}$, Hill D, et al. Repetitive mass practice or focused precise practice for retraining walking after incomplete spinal cord injury? A pilot randomized clinical trial. Neurorehabil Neural Repair. 2014;28:314-24.

48. Kraemer WJ, Adams K, Cafarelli E, Dudley GA, Dooly C, Feigenbaum MS, et al. American College of Sports Medicine position stand. Progression models in resistance training for healthy adults. Med Sci Sports Exerc. 2002;34:364-80. 